\title{
LA MÉTRICA EN EL REFRANERO ESPAÑOL
}

THE METRIC IN SPANISH PROVERBS

\author{
LA MÉTRIQUE DANS \\ LES PROVERBES ESPAGNOLS
}

\author{
ARCADio PARdo \\ Université Paris X Nanterre
}

Resumen: Los refranes se expresan en formas métricas. Se explora en este trabajo su diversidad en cuanto a versos, rimas y formas estróficas, así como el parentesco de los refranes con las canciones de tipo tradicional. Los refranes tienen en muchos casos su origen en la sabiduría bíblica e incluso en civilizaciones anteriores. Y se encuentran igualmente en la tradición de civilizaciones ajenas a la tradición occidental.

Palabras clave: refranes, métrica, verso solo, verso aislado, canciones, Eclesiastés, sabiduría, proverbios, Sem Tob.

Abstract: Statements separated from the rest of a text (isolated verse, title, proverb) can be submitted to a metrical anaysis. Besides, the title of many plays are proverbs and such notions as verse, rhyme (nature and distribution) must be taken into account in order to characterize the metrical structure of a proverb. In this aspect, the Spanish proverb has an ancient tradition, going back to the sapientals texts of the Bible. The popular wisdom then becomes integrated in an autochtonous metrics without losing its universality. 
Keywords: proverbs, metrics, verse, rhyme, stanza, sapiential formulas.

Résumé: Les proverbes s'expriment selon des formes métriques. On explore dans ce travail leur diversité en matière de vers, de rime, de forme strophique et la parenté des proverbes avec les chansons de type traditionnel. Les proverbes ont souvent la sagesse biblique pour origine quand il ne faut pas remonter à des civilisations plus anciennes encore. On les retrouve également dans des civilisations étrangères à la tradition occidentale.

Mots-clés: proverbes, métrique, vers simple, vers isolé, chanson, Ecclésiaste, sagesse, proverbes bibliques, Sem Tob. 
No hay refrán que no sea verdadero. Horozco, Teatro universal de proverbios, 2056.

$\mathrm{E}$

n la mayoría de los refranes citados en las páginas que siguen se ha señalado su procedencia (Horozco, Canellada, etc.). En algunos casos se cita la procedencia indicando además su localización en la recopilación hecha por Felipe C. R. Maldonado.

Con pocas excepciones, los refranes citados en este trabajo proceden directamente de las obras siguientes:

- Sebastián Horozco, Teatro universal de proverbios, ed. de José Luis Alonso Hernández, Salamanca, Ediciones Universidad de Salamanca, 1986.

- Luis Martínez Kleiser, Refranero general ideológico español, Madrid, Real Academia Española, 1953.

- Felipe C. R. Maldonado, Refranero clásico español, Madrid, Taurus, 1966. Este conjunto antológico ofrece una selección de los contenidos en las obras del Marqués de Santillana, Blasco de Garay, Pedro Vallés, Hernán Núñez, Juan de Mal Lara, Sebastián de Horozco y Gonzalo Correas.

- María Josefa Canellada y Berta Pallares, Refranero español. Refranes, clasificación, significación y uso, Madrid, Editorial Castalia, 2001.

Cabe señalar los interesantes trabajos de Jesús Cantera Ortiz de Urbina: «El refranero judeoespañol» ${ }^{1}$, así como Refranes, otras paremias y fraseologismos en Don Quijote de la Mancha ${ }^{2}$.

${ }^{1}$ Cantera Ortiz de Urbina, Jesús, «El refranero judeoespañol», Paremia, 6 (1997), pp. 153-162.

2 Cantera Ortiz de Urbina, Jesús, Refranes, otras paremias y fraseologismos en Don Quijote de la Mancha, Vermont, University of Vermont, 2005. 
En mi trabajo «Verso aislado, verso solo, verso-poema», publicado en Rhythmica ${ }^{3}$, propuse se considerasen poemas aquellos versos que aparecen aislados en página e independientes de los textos anteriores o posteriores de la edición. Un ejemplo puede ser este de Carlos E. de Ory:

Humanos son los pájaros también ${ }^{4}$.

O los numerosos del mismo tipo que se encuentran en la obra de Alberti, aunque no aparezcan siempre aislados en página sola, como estos que siguen:

Cada mañana, el mar echa los dientes ${ }^{5}$.

En su libro $A$ la pintura, se encuentran numerosos versos de este tipo:

¿Cuántos azules dio el Mediterráneo? ${ }^{6}$

Y algunos de extremada concisión:

El verde solitario de la muerte ${ }^{7}$.

Estos versos que aparecen como ensalzados en su soledad, inducen a recordar otros enunciados en su forma sintáctica escueta que encontramos, como si fueran lemas, en algunos títulos de obras de teatro:

La vida es sueño (Calderón de la Barca)

Quien calla, otorga (Tirso de Molina)

O con formas sintácticas en las que algún elemento, generalmente el verbo, se ha eludido, aportando así una mayor contundencia a la expresión:

Del rey abajo, ninguno (Francisco de Rojas Zorrilla)

El mejor alcalde, el rey (Lope de Vega)

El árbol del mejor fruto (Tirso de Molina)

3 PARDo, Arcadio, «Verso aislado, verso solo, verso-poema», Rhythmica, 2 (2004), pp. 201-233.

${ }^{4}$ OrY, Carlos Edmundo de, Lee sin temor, Madrid, Editora Nacional, 1976, p. 202.

5 Alberti, Rafael, Poesía [1924-1944], Buenos Aires, Editorial Losada, 1946, p. 292.

6 Alberti, Rafael, A la pintura, Buenos Aires, Editorial Losada, 1953, p. 33.

${ }^{7}$ Ibid., p. 84. 
Pueden encontrarse igualmente en títulos de obras no dramáticas como es el caso de este de una de las leyendas de Zorrilla:

A buen juez, mejor testigo (José Zorrilla).

El parentesco entre este tipo de expresiones y muchos refranes castellanos es evidente. Véanse estos que siguen:

- Con elisión del verbo:

Hombre mendigo, nunca buen testigo (Correas, 1487 en Canellada y Pallares).

- O con mayor concisión:

En abril, / aguas mil (Horozco, 11).

Pueden ponerse en paragón también, por ejemplo, el título de la leyenda de Zorrilla y los refranes que citamos ahora:

A buen juez, mejor testigo (José Zorrilla).

A pan duro, diente agudo (Santillana, 2525 en Canellada y Pallares).

Significan que siempre hay alguien que supera las cualidades ya de por sí meritorias (el buen juez), o algo que conviene para resolver un trance delicado (el diente agudo).

- O estos dos, el primero de los cuales ya citado:

Del rey abajo, ninguno

Amor y rey no sufren ley (Martínez Kleiser, 3730).

Títulos de obras y refranes pueden tener realización semejante o próxima en su expresión sintáctica. Estos que siguen tienen identidad formal:

La vida es sueño

El tiempo es oro (Martínez Kleiser, 60600).

Numerosos serán los refranes que expresan identidad en sus términos enlazados por el verbo ser:

Mal vecino es el amor (Horozco, 1752).

La nobleza es antigua riqueza (Martínez Kleiser, 45631).

Uno muy conocido ha sido utilizado íntegramente como título por Miguel Ramos Carrión (1848-1915) que fue también autor de 
zarzuelas (Agua, azucarillos y aguardiente) y de la comedia que interesa aquí, titulada

Más vale tarde que nunca, proverbio original en un acto y en prosa.

En este título, el calificativo de original debe referirse a esta obra pues el proverbio aparece ya en el Teatro universal de proverbios de Sebastián de Horozco con el número 1815.

Tirso de Molina da a una de sus comedias el nombre de Tanto es lo de más como lo de menos, que es refrán recogido por Sbarbi e incluido en el Refranero de Canellada y Pallares con el número 2812. Y no cabe duda de que otros títulos suyos como los siguientes Quien no cae, no se levanta (Horozco, 2515) y No le arriendo la ganancia (Correas, 1146 en Maldonado) proceden del corpus tradicional.

Esta «consanguinidad» de proverbios y títulos de obras literarias se centra muy especialmente en las relaciones entre proverbios y soluciones métricas en su diversidad de metros, rimas, formas estróficas. La parcelación de un refrán en versos ya viene señalada por las rimas, aunque a menudo esa parcelación no parezca evidente cuando versos sueltos forman parte del conjunto. Sebastián de Horozco cortó los miembros con un trazo /, pero no siempre su división coincide con las formas naturales del verso. Se ha tratado aquí de restituir, cuando el error es evidente, la separación lógica de los miembros.

Por otra parte, puede ocurrir que un mismo texto aparece a la vez en recopilaciones de proverbios y en muestras de poesía tradicional idénticos o con alguna variante, como estos dos ejemplos:

Esta novia se lleva la flor que las otras no (Correas, 821 en Maldonado).

Reaparece en

Este niño se lleva la flor,

que los otros no (Lope de Vega, El piadoso aragonés)

Así como:

Entra mayo y sale abril; cuán floridito le vi venir (Correas, 782 en Maldonado).

Entra mayo y sale abril, tan garridico le vi venir ${ }^{8}$. ${ }^{8}$ Alonso, Dámaso, y Blecua, José María, Antología de la poesía de tipo tradicional,
Madrid, Gredos, 1956, 30 . 
O el tan común

Todo tiempo pasado

fue mejor (Correas, 1370 en Maldonado)

que mucho antes que en Manrique ya se encuentra en pasajes bíblicos como en esta pregunta: «¿Por qué es que los tiempos pasados fueron mejores?» que se encuentra en Eclesiastés, 7-9.

Se observan a continuación las formas métricas en que se expresan los refranes generalmente coincidentes con las formas tradicionales: verso, rima y estrofa.

\section{El verso}

Todo refrán tiene su realización en verso y así lo recibe el lector o el recitante, aunque es de señalar la frecuencia en versos de distintos tipos. Julio Casares clasificó los refranes, como es sabido, en unimembres, bimembres y plurimembres, señalando la preponderancia de los del segundo tipo: «La forma usual del refrán es bimembre, o, por amplificación de dichos conceptos, plurimembre» ${ }^{9}$ : - Unimembres son algunos de los arriba citados: La vida es sueño / Mal vecino es el amor. (Horozco, 1756).

- El miembro de una sola sílaba puede existir aunque sea rarísimo, y como tal verso contaría dos sílabas.

- En los conjuntos bimembres y plurimembres, los refranes respetan o no la igualdad de medida de los versos. Tratándose de pareados, se observa que los dos miembros tienden a igualar su forma métrica, aunque abundan los casos de disimetría. En refranes plurimembres hay una amplia variedad de versos que forman un conjunto estrófico con también muy amplia variedad. Se recogen ahora algunos de los múltiples casos.

- El miembro de dos sílabas se detecta en refranes como este:

Uno / y ninguno, / todo es uno (Núñez, 238 en Maldonado).

Suegra, / ni de barro buena (Horozco, 2880).

- Trisílabo: Carga cerrada / o puede ser algo / o nada (Horozco, 527).

Guárdate, / y guardarte he (Horozco, 1232).

— Tetrasílabo: Boca que dice no, / dirá sí (Horozco, 444).

Casa vieja, / toda va tras una teja (Horozco, 535).

${ }^{9}$ CASARES, Julio, Introducción a la lexicografia moderna, Madrid, R.F.E., Anejo LII, 1969, p. 192. 
- Pentasílabo: Cierra tu puerta / y alaba a tu vecino (Horozco, 549).

En río manso, no metas tu mano

(Correas, 773 en Maldonado).

— Hexasílabo: Todo es menester, / migas y sorber (Horozco, 2962).

Vieja escarmentada, / pasa el agua arregazada

(Horozco, 3063).

- Heptasílabo: De tales romerías, / tales venerías (Horozco, 747).

No dice más la lengua, / que lo que piensa la cabeza

(Martínez Kleiser, 20.258).

- Octosílabo: Del que vergüenza no tiene / toda la villa es suya

(Horozco, 674).

Oye mucho y habla poco, / pues lo contrario hace el loco

(Martínez Kleiser, 29.311).

Se encuentran miembros de sílabas superiores. Aunque nada frecuente, se señala este endecasílabo que sigue como curiosidad:

Quien da lo suyo antes de su muerte, / que le den con una porra en la frente (Horozco, 2586).

\section{La rima}

Aunque existen bimembres y plurimembres de componentes sueltos, los refranes se sostienen generalmente por la rima que, como en el verso integrado en el poema, presenta diversas modalidades. Se exponen las siguientes:

A. Rima oxitona en vocal libre:

El que malas mañas ha, / tarde o nunca las perderá (Horozco, 1000).

El cantar que no sé, / cómo lo diré? (Horozco, 885).

Menosprecia al rey / el que no guarda su ley (Horozco, 1874).

$\mathrm{Al}$ revés me la vestí, / ándese así (Horozco, 136).

Lo poco mucho duró, / y lo mucho se gastó (Correas, 956 en

Maldonado).

No se han encontrado proverbios rimados en final ú. Margit Frenk recoge uno del Cancionero musical de Palacio que merece, por su rareza, que se cite aquí:

Cucú, cucú,

iguarda no lo seas tú! (CMP, 94) ${ }^{10}$.

${ }^{10}$ Frenk, Margrit, «Sobre los cantares populares del Cancionero musical de palacio», Anuario de Letras: Lingüística y filología, vol. 35 (1997), pp. 215-235, p. 226. 
B. Rima oxitona en vocal trabada:

Asiéntate en tal lugar / que nadie te pueda echar (Horozco, 66).

A la vejez / los aladares de pez (Horozco, 103).

Se nace para morir; / se muere para vivir (Martínez Kleiser, 42.252).

Las palabras buenas son, / si así es el corazón (Correas, 1188 en Maldonado).

Tampoco se han encontrado con final en ú trabada. En este que se cita aparece como final de su primer miembro pero quedando sueltos los dos que lo componen:

Lo que has de dar al mur, / dalo al gato (Horozco, 1590).

Citamos en cambio este que sigue porque ofrece un ejemplo de rima dificultosa que bien pudiera hacer sospechar un origen culto:

Quien sus carros unta / sus bueyes ayunta (Horozco, 2578).

C. Rimas en terminación paroxitona:

Naturalmente la rima en palabras llanas es la más frecuente:

Buena memoria es la escritura; / ella retiene bien su figura

(Correas, 220 en Maldonado).

Y se encuentra una muy extensa variedad.

D. Rimas proparoxitonas:

El final esdrújulo es de muy escasa frecuencia. Tras búsqueda paciente se han recogido estos:

A la hija mala, / dale dinero y cásala (Canellada y Pallares, 1409).

Picóme una araña / y atéme una sábana (Canellada y Pallares, 230).

Naturalmente, se encuentran finales de miembros esdrújulos pero como versos sueltos. He aquí algunos:

Abrazos y besos / no hacen chiquillos, / pero tocan a vísperas

(Canellada y Pallares, 17).

Alegrías, albardero, / que se quema el bálago (Horozco, 130).

Cuando te dieren un gobierno, / cógele (Canellada y Pallares, 1269). 
Un caso curioso es el que sigue en el que el esdrújulo final viene como eco de rimas internas:

Hombres desleales, mujeres sin vergüenza, mar sin peces y bosques sin leña: eso es Génova. (Canellada y Pallares, 1547).

Aunque no el único. Recuérdense estos en los que el refrán se apoya en la sucesión de ecos fonéticos:

Faldas y cartas mandan en España (Martínez Kleiser, 22.977).

Espera, Pérez, y no desesperes (Martínez Kleiser, 22.701).

A lo hecho, ruego y pecho (Correas, 79 en Maldonado).

Madre pía, mala hija cría (Horozco, 1703).

Vivir, servir y pedir hacen a los hombres subir (Correas, 1423 en Maldonado).

Dolor de codo, dolor de esposo; duele mucho y dura poco (Vallés, 34 en Maldonado).

La rueda de la fortuna nunca es una (Núñez, 151 en Maldonado).

\section{E. Distribución de la rima:}

Los refranes bimembres pueden presentar las siguientes posibilidades en cuanto se refiere a la rima. Señala Berta Pallares como una de las características del refrán tipo (apartado g), que «el final del grupo 2 y del grupo 3 van realzados por una rima perfecta» ${ }^{11}$. Se refiere, naturalmente, a refranes plurimembres de tres miembros, y se entiende que su rima perfecta es la rima consonante.

El esquema de las rimas en los refranes bimembres puede ser el siguiente:

1) Los dos miembros no rimados:

Dar es señorío, / recibir es servidumbre (Correas, 821 en Canellada).

2) Primer miembro con final oxítono $y$ segundo suelto:

Por mucho madrugar / no amanece más aína (Martínez Kleiser, 3857)

3) Primer miembro suelto y segundo miembro oxítono:

Cuan lejos de ojos, / tan lejos de corazón (Martínez Kleiser, 4141).

4) Ambos miembros rimados con final oxítono:

Del mirar nace el amar / y del no ver el olvidar (Martínez Kleiser, 4148).

${ }^{11}$ Canellada, María Josefa, y Pallares, Berta, Refranero español. Refranes, clasificación, significación y uso, Madrid, Editorial Castalia, 2001, p. 18. 
5) Ambos miembros rimados con final paroxítono

Más vale pájaro en mano / que buitre volando (Santillana, 2512 en Canellada).

Una evaluación de frecuencias, sin pretensiones de absoluta veracidad, inclina a afirmar que el tipo más utilizado es el 4, o sea con ambos miembros oxítonos rimando entre sí; en cambio el tipo 2 parece ser el menos utilizado. María Josefa Canellada hace una estimación de frecuencia de rimas y señala la primacía de la rima consonante sobre la asonante ${ }^{12}$.

Naturalmente, la variedad es grande, habiéndolos también con rimas internas consonantes en un miembro y asonantes en otro, como en este:

Obeja y abeja y piedra que trebeja (Horozco, 2260).

\section{F. La estrofa:}

Los refranes no nos han llegado -o no nos han llegado siempreen su apariencia métrica. Horozco los presenta separando, como ya se ha dicho, los versos con un trazo, pero la división propuesta no siempre coincide con la lógica, y en otros refraneros aparecen en frases como prosa. Los que se presentan aquí se han parcelado en versos según la lógica de su significado.

«Ay también en los refranes rhytmo, que es una manera de cantar», escribió Juan de Mal Lara en el siglo XVI ${ }^{13}$. Que viene a significar que en los refranes hay formas estróficas más o menos semejantes a las de los cantares o coplas, como ya lo reconocieron entre otros Alfonso Reyes: «El aire de canción de algunos proverbios es la única explicación de su existencia» ${ }^{14}$ y Henríquez Ureña: «Muchos de los viejos adagios españoles son dísticos de metro regular o irregular con rima consonante o asonante ${ }^{15}$. Y también Unamuno en su Cancionero, $\mathrm{n}^{\mathrm{o}} 395$ :

en sentencias cuajó sabiduría prontas a la canción.

I2 Ibid., p. 435.

${ }^{13}$ Mal Lara, Juan de, Philosopha Vulgar, Sevilla, 1568. Citado por Canellada, María Josefa y Pallares, Berta, Refranero español, cit., p. 420.

${ }^{14}$ ReYEs, Alfonso, De los proverbios y sentencias vulgares. Obras completas, México, Fondo de Cultura Económica,1955, p. 169.

15 Henríquez Ureña, Pedro, Versificación irregular española, Madrid, ed. Hernando, 1933, p. 92. 
Debe recordarse a este propósito cómo Santillana integra en el Villancico que hizo a sus tres hijas, cuatro cancioncillas tradicionales que son las siguientes:

Aguardan a mí, / nunca tales guardas vi.

La niña que amores ha, / sola, cómo dormirá?

Dejadlo al villano pene: / véngueme Dios dele.

Sospirando iba la niña / e non por mí, / que yo bien se lo entendí16

Se contemplan en este apartado los refranes de dos o tres miembros dado que los de cuatro miembros pueden asimilarse a cuartetas de medida y rima variables pero fácilmente reconocibles. $\mathrm{O}$ bien por unión de dos pareados como en este ejemplo:

Por dinero / baila el perro, / y por pan / si se lo dan.

La semejanza de las formas estróficas de los refranes con estrofas tradicionales es grande. Se encuentran conjuntos muy variados en cuanto a metros y disposición de rimas. Los miembros del refrán pueden presentar cierta uniformidad en la medida de los versos, pero también diversidad: un verso corto y dos superiores en sílabas, o dos versos cortos y uno más largo, o bien identidad en el tipo de versos.

El parentesco entre refranes y canciones se manifiesta en la semejanza de formas métricas. Se han escogido aquí algunos refranes $\mathrm{y}$ algunas canciones con semejanza de formas y disposición de las rimas aunque no siempre en total identidad. Los que siguen deben de ser muestra suficiente:

$$
\text { Refranes }
$$

\section{Canciones}

- Pareado:

Otro vendrá

que bueno me hará.

(Correas, 1180 en Maldonado)
Dime, señora, di, si te acordarás de $m i^{17}$.

- Verso suelto y dos rimados:

Ata corto y piensa largo,

Ojos morenicos, procura herrar somero si quieres ir caballero. (Horozco, 365)

irm'e yo a querellar

que me queredes matar ${ }^{18}$.

${ }^{16}$ SAntillana, Marqués de (Íñigo López de Mendoza), Serranillas, Barcelona, Planeta, 1988, p. 17.

17 Alonso, Dámaso, y Blecua, José María, Antología, cit., p. 339.

18 Cancionero musical de Palacio, ed. de Joaquín González Cuenca, Madrid, Visor libros, 1996, p. 335. 
- Versos 1 y 3 aconsonantados y verso 2 suelto:

Todo te haré, mas casa con dos puertas no te guardaré. (Santillana, 163 en Maldonado)
Pues mi pena veis, miradme sin saña, o no me miréis ${ }^{19}$.

- Octosílabos; el 1 suelto y 2-3 en pareado:

Adonde el maravedí se dejó de ti hallar debes otro allí buscar. (Horozco, 56)

Vuestros ojos morenillos, que por mi desdicha vi, me hacen vivir sin $m i^{20}$.

- Dos tetrasílabos y un octosílabo aconsonantados:

Cordobés, mala res;

A ti amo,

de una aguja hace tres.

a ti llamo,

(Vallés, 22 en Maldonado)

porque eres el verde ramo ${ }^{21}$.

- Pareado inicial y verso suelto:

A la tercera

En Ávila del Río

buena y verdadera, a tres va la vencida.

(Horozco, 118) mataron a mi amigo, dentro de Avila ${ }^{22}$.

- Tres octosílabos: 1 suelto; 2 y 3 en pareado:

Caballo ligero en guerra, hombre de armas en paz, infante nunca jamás. (Correas, 241 en Maldonado)

Aquel caballero, madre tres besicos le mandé: creceré y dárselos he ${ }^{23}$.

\section{- Tres versos rimados:}

Ama a quien no te ama, responde a quien no te llama: andarás carrera vana.

No puedo apartarme de los amores, madre, (Correas, 91 en Maldonado). no puedo apartarme $e^{24}$. (Cancionero musical de Palacio, 361).

\footnotetext{
$* * *$

${ }_{19}$ Alonso, Dámaso, y Blecua, José María, Antología, cit., p. 9.

${ }^{20}$ Ibid., p. 39.

21 PéRez de Guzmán, Fernán, Cancionero Castellano del siglo XV, Madrid, Bailly-Baillière, 1912, pp. 700 y 302.

${ }^{22}$ Alonso, Dámaso, y Blecua, José María, Antología, cit., p. 38.

${ }^{23}$ Ibid., p. 21.

${ }^{24}$ Cancionero musical de Palacio, cit., p. 361.
} 
De este sondeo somero en el amplio territorio de los refranes (65.083 en la recopilación de Luis Martínez Kleiser) se puede concluir recordando la convicción ya declarada por los más sabios estu$\operatorname{diosos}^{25}$, de que los refranes pueden considerarse, desde el punto de vista de sus formas diversas, como expresiones que se realizan en formas métricas, tanto en cuanto a versos como a rimas y estrofas.

Parece evidente, aunque no ha sido el propósito de este trabajo y que sabios entendidos ya lo hayan señalado, que los refranes tienen precedentes muy antiguos en la literatura moral y en particular en el Libro de Proverbios y el Eclesiastés del antiguo Testamento. Ya desde su principio, la Exhortación al estudio de la sabiduría, en el primero de los citados (Escucha, hijo mío, la instrucción de tu padre...) llama la atención el eco de estas palabras que resuenan en los primeros versos de Santillana:

Fijo mío muy amado,

para mientes

e non contrastes las gentes

mal su grado,

ama e serás amado ${ }^{26}$.

Y no sorprende encontrar proverbios cuya forma sintáctica -salvando las distancias del tiempo y de la lengua- recuerda la de los refranes en su forma actual recibida del pasado.

La sabiduría moral se ha vertido desde tiempos remotos en formas breves relacionadas con ritmos y formas emparentadas con las formas métricas autóctonas que han encontrado prolongación en poetas en nuestras lenguas actuales. He aquí algunos ejemplos:

Una forma de premonición con expresión de condición y consecuencia abunda ya en la sabiduría mesopotámica en formas bimembres:

Si una mujer da a luz un cojo, / habrá calamidades en su casa

(Sentencia mesopotámica) ${ }^{27}$.

que tiene paralelismo semejante en este refrán castellano:

$\overline{25}$ Pueden consultarse entre otros, además de los citados en la nota 5, los siguientes: CASARES, Julio, Introducción a la lexicografía, cit.; CEJADOR, Julio, Refranero castellano, Madrid, Analecta, 2008; FrenK, Margrit, «Sobre los cantares populares», cit.; O'KANE, Eleanor S., Refranes y frases proverbiales españolas de la edad media, Madrid, Anejos del Boletín de la Real Academia Española, 1959.

26 Santillana, Marqués de (Iñigo López de Mendoza), Proverbios o Centiloquio, Barcelona, Planeta, 1988, p. 122.

${ }^{27}$ Bottéro, Jean, La plus vieille religion en Mésopotamie, Paris, Gallimard, 1998, p. 338. 
No te acuestes con tu sirvienta, / te llamará Canalla ${ }^{28}$.

De interés es el largo poema de Sem Tob Glosas de Sabiduría o Proverbios morales en los que Agustín García Calvo detecta la herencia de la tradición profana y de la tradición bíblica: «De la Biblia [...] lo son sobre todo (los lugares de donde proceden) de los Proverbios y el Eclesiastés $\gg{ }^{29}$. Y se encuentran en el refranero algunos que bien pudieran ser eco de lo mismo:
Nin [..] pued'omr alcançar cosa
Cada cosa que ves /
sinon con su revés ${ }^{30}$. tiene su haz y su envés. (Martínez Kleiser, 4509).
Quien antes non esparze
Echa tierra sobre tierra trigo, non lo allega; si so la tierra non yaze, a espiga non llega ${ }^{31}$.

Es de justicia recordar que ya Unamuno resaltó la herencia bíblica recogida por Sem Tob:

Lamentos de las entrañas de Job, visiones de la escala de Jacob, se perlan en los proverbios de Sem Tob

Son versos que se encuentran en su Cancionero con el número $1255^{32}$.

Podrían multiplicarse los ejemplos de semejanzas en su realización entre las formas antiguas que se expresan en fórmulas bimembres o plurimembres, y las bíblicas. He aquí otros ejemplos en los que se percibe como un eco en los contenidos como en estos que siguen:

Como nube pasó mi ventura.

La mayor ventura menos dura.
Job, 30-15.

(Martínez Kleiser, 59.487).

Pues la sabiduría vale más que las perlas, y cuanto hay de codiciable no puede comparársele.

Proverbios, 8-22.

\footnotetext{
$\overline{28}$ Ibid., p. 224.

29 García Calvo, Agustín, Don Sem Tob, Glosas de Sabiduría o Proverbios morales y otras Rimas, Madrid, Alianza editorial, 1974, p. 22.

${ }^{30}$ Ibid., vv. 447-448, p. 63.

31 Ibid.

32 Unamuno, Miguel de, Cancionero, Madrid, Akal editor, 1984, p. 417.
} 
Más vale el sabio con sus letras que el rico con sus talegas.

Lo que fue, eso será. Lo que fue es y será.
(Martínez Kleiser, 56.885).

Eclesiastés, 1-8.

(Horozco, 1643).

No hay memoria de lo que precedió, ni de lo que sucederá habrá memoria en los que serán después.

Lo que fue y no es ya, menos es que lo que será.

Eclesiastés, 1-11

(Martínez Kleiser, 48.948).

Todo esto confirma la ascendencia antigua o bíblica global de los refranes.

Otras culturas sin trabazón con las antiguas evocadas, han condensado también esa sabiduría en sentencias sorprendentemente semejantes a las que nos son familiares. He aquí algunas africanas:

No puedes esconder el humo si encendiste fuego

que se dice en Burundi y tiene la estructura condición-consecuencia, aunque aquí invertida. O este otro de estructura regular y mensaje esperanzador:

Recuerda, si hay tormenta, habrá arco iris.

Igual sabiduría ofrecen los refranes en las culturas amerindias. Véase este que sorprende por su semejanza con expresiones muy próximas a algunas de los libros sapienciales bíblicos:

Nosotros no heredamos la tierra de nuestros ancestros; sólo la tomamos prestada de nuestros hijos.

\section{Conclusión}

Lo anteriormente expuesto induce a concluir que no parece aventurado afirmar que todo tipo de proverbio en cualquiera de sus contenidos (consejo, sentencia, etc.) y en cualquiera de las culturas dispersas por los continentes se hermanan en sus expresiones formales de lo que puede llamarse métrica autóctona y que su estudio obliga a considerarlos, además de desde su contenido moral, como expresiones que revelan una innegable universalidad e incluso una consanguinidad por encima de relaciones en el tiempo y en el espacio que todavía ignoramos. 